\title{
Social Facilitation of Dominant and Subordinate Responses ${ }^{1}$
}

\author{
Robert B. Zajonc and Stephen M. Sales \\ The University of Michigan
}

\begin{abstract}
The hypothesis was examined that, because it is drive-producing, the presence of an audience enhances the emission of dominant responses and inhibits the emission of subordinate responses. Thirty-nine subjects performed a pseudo-recognition task in which their guessing responses were based on dominant and subordinate habits, previously established by means of differential training. The probability of dominant responses was found to be higher for subjects working in the presenee of an audience than for those working alone. The opposite result, however, was observed for subordinate responses. These findings are related to others in the area of social facilitation.
\end{abstract}

Social facilitation studies show that the presence of other organisms, as coactors or as spectators, enhances performance on such tasks as multiplication (Allport, 1924; Dashiell, 1930), chain association (Allport, 1924), pursuit rotor (Travis, 1925), signal detection (Bergum and Lehr, 1963), etc. The eating response, too, has been observed to increase in the presence of others (Harlow, 1932; James, 1953; Tolman and Wilson, 1965). Some studies, however, seem to show that the presence of other organisms has detrimental effects. The presence of spectators, for instance, was found to interfere with nonsense-syllable learning (Pessin, 1933) and with finger-maze learning (Husband, 1931). Animal subjects were also observed to suffer interference in maze learning when other members of the same species were present (Gates and Allee, 1933; Klopfer, 1958).

These seemingly conflicting experimental results are reconciled by assuming that the presence of others has arousal consequences (Zajonc, 1965). If this assumption is valid, we would expect the presence of others to manifest the same pattern of effects as are obtaincd by increasing generalized drive (D) state, such as, for instance, the enhancement of dominant responses (Spence, 1956). If for a given experimental task dominant responses are largely correct ones-as they are in the performance of previously acquired skills-then the presence of others will

\footnotetext{
${ }^{1}$ This research was supported in part by the Office of Naval Research, Contrant Nonr-1224 (34) 170-309, and by the National Science Foundation, grant GS-629.
} 
result in a better performance. If, howerer, dominant responses are largely incorrect-as they are, for instance, in the early stages of learning a maze-then the presence of spectators or of coactors will delay the acquisition of correct responses by enhancing the emission of the incorrect ones.

Some recent physiological evidence is consistent with the assumption that the presence of other organisms is a source of arousal. This evidence shows that their presence is associated with increased adrcnal (Thicssen, 1964) and adrenocortical activity (Mason and Brady, 1964) - both fairly reliable indices of general arousal level.

The purpose of this study is to examine the effects of an andience on the emission of dominant and subordinate responses. A procedure previously found sensitive to drive effects was employed. In a study of word recognition Zajonc and Nieuwenhuyse (1964) established competing verbal habits, varying in strength, by exposing subjects to different verbal stimuli different numbers of times. After training, these verbal stimuli served in a recognition and pseudo-recognition task. On recognition trials the verbal stimuli were presented tachistoscopically for purposes of threshold assessment. Interspersed among these trials were others (pseudo-recognition trials) on which the subject was led to believe that a stimulus was actually shown, while only a rapid flash of an empty tachistoscope was presented to him. Since subjects were instructed to guess on every trial what verbal stimulus was shown, and since no stimuli were present on these pseudo-recognition trials, their guessing responses on these trials were alone a function of the habits established in training. Because on any one trial the subject could make only one out of several alternative responses, these habits were in competition with each other, and we can, therefore, speak of the strong habits as dominant, and of the weak habits as subordinate. A group working under aroused drive was compared with a low-drive group for emission of pseudo-recognition responses. The results were entirely consistent with the prediction derived from Spence's (1956) theory of drive effects: under increased drive dominant responses were enhanced and subordinate responses were attenuated. The overall effect manifested itself in an interaction between the habit-strength and drive variables.

If the presence of others has indeed arousal consequences similar to those of drive (D), then the above effect should also be obtained when the direct motivational manipulation in the Zajonc-Nieuwcnhuyse experiment is replaced by manipulating the presence of spectators. It is predieted, thercfore, that in the present study response cmission in the pseudo-recognition task will be characterized by an interaction between the habit-strength and the audience-variables. 


\section{METHOD}

\section{Subjects}

The subjects were 39 male students drawn from the University of Michigan subject pool. Twenty were assigned at random to one condition (Control) and 19 to another (Facilitation): Five subjects in the first condition and four in the second were discarded for failing to learn more than three response words. The subjects' ages ranged from 18 to 24 . Each subject was paid $\$ 1.25$ for participating in the experiment.

\section{Stimulus Materials}

The verbal stimuli were ten of the "Turkish words" (seven-letter nonsense words) used in the recognition studies of Solomon and Postman (1952) and Zajonc and Nieuwenhuyse (1964). Slight changes in some spellings were made to facilitate pronunciation. The ten words were printed in large black letters on white paper and then photographed. These photographs, each $4 \times 6$ inches, were used in the training session. Black-and-white $2 \times 2$ inch slides, made from the photographs, were used in the testing session.

\section{Procedure}

Training procelure. The ten stimulus words were divided into five trainingfrequency classes $(1,2,4,8$, and 16$)$, each including two words. The training frequencies and words were counterbalaneed so that cach word was used in every frequency class equally often. Each counterbalanced order was given to six subjects, three in each condition.

Subjects were run individually. Upon entering the laboratory the experimenter instructed the subject that the experiment consisted of two parts, the first being concerned with learning to pronounce foreign words. Without saying anything about the second part, the experimenter proceeded with the training. For each subject a random order of stimulus presentation was previously arranged. The entire training session consisted of 62 presentations (two verbal stimuli in each of the five frequency classes). On each presentation the experimenter would show the stimulus, pronounce it aloud, and then have the subject repeat it once. The subject's response was neither reinforced nor corrected. An interval of approximately four seconds separated the presentations.

Pseudo-recognition test. After the completion of training the experimenter told the subject that the second half of the experiment dealt with subliminal perception, The subject was told that the foreign words he had just learned to pronounce would be flashed upon a small screen and that the speeds and illumination at which they would be flashed would on most trials make their recognition impossible. The subject's task, however, would be to say what word was shown on each presentation, even when there was rery little to see. In these cases the subject was told he would simply have to guess.

For the pseudo-recognition test the subject was left alone in his cubicle. Adjacent to this cubicle was the experimenter's projection room, equipped with a shutterprojector and an intercom system to the subject's cubicle. Stimulus slides were flashed through a one-way mirror between the cubicles. The lights in the subject's cubicle were out, although some light from the experimenter's projection room 
provided dim illumination for the subject. Subjects were given to understand that the experimenter could not see them during the pseudo-recognition session.

The stimulus slides were presented in four blocks of 41 presentations each. Within each block ten randomly interspersed presentations involved showing one each of the ten verbal stimuli used in training. They were flashed all 1/u second, with at medium diaphragm opening. 'The speed and diaphragm setting were selected prior to the experiment proper so that they would result in about $90 \%$ correct recognition. The remaining 31 presentations were made at $1 / 100$ second and with an extremely small diaphragm opening. At these settings recognition, as determined in pretests, was a matter of chance. Shown on each of these 31 presentations was a slide of the same size and with a similar configuration as the ten verbal stimulus slides. However, irregular black lines were drawn on these slides in the place of letters. Five different pseudo-stimulus slides were used for this purpose, alternating randomly among the 31 pseudo-recognition presentations. There was a 10 -second interval between trials. Trial blocks were also separated by a 10 -second interval.

To feedback except as noted below on either the ten real slides or on the 31 psido-stimulus slides was given, nor was there any form of reinforcement.

Responses were recorded by the experimenter as they were made by the subject. In ases when the response was not identical or cjuite close to any of the ten stimulus words, the response was transcribed phonetically and a decision was made after the session concerning which word was meant by the subject. In general, errors in pronunciations were made on the second or third syllables. The ambiguous responses wore therefore classified as one or the other stimulus words according to the first syllable. On three occasions a subject's response bore no resemblance whatever to any stimulus word. In these cases the subject was told that this was not one of the foreign words he learned, and was asked to make another guess. This was the only form of feedback ever provided.

Experimental conditions. After training and after the experimenter issued instructions for the pseudo-recognition trials, subjects in the Control condition were given a. copy of Time to read aloud (ostensibly, to adiust the intercom system). This interpolated activity was intended to prevent the subjects from rehearsing the nonsense words, and it took approximately 1 minute. The entire pseudo-recognition session was conducted with the subject alone in his cubicle and obviously out of the experimenter's sight.

After having received instructions for the pseudo-recognition series, subjects in the Facilitated condition were told that two students of the experimenter had asked if they could watch the experiment and that they would be with the subject during the remainder of the session. The "students"-always strangers to the subject-were then introduced by the experimenter to the subjert's cubicle and were seated a few feet away. The students did not talk with each other or with the subject. They merely watched the subject, without, however, reacting differentially to his responses. The subjects in the Facilitated condition also were asked to read from Time for 1 minute to inhibit rehearsal.

\section{RESULTS}

The overall effect of audience on responses of different strengths is shown in Fig. 1. The data in this graph represent the subjects' responses on the 124 pseudo-recognition trials (four blocks of 31 trials each). The average number of responses per trial block is plotted on a log scale 


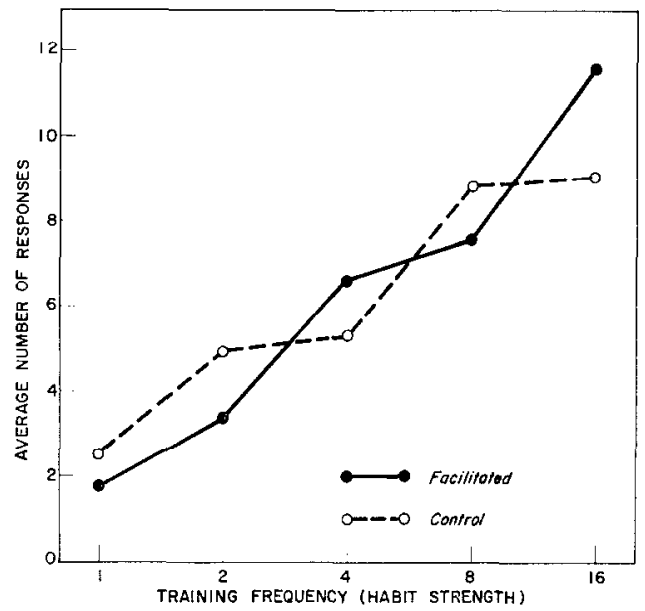

Fig. 1. Number of responses of different frequency classes emitted during the pseudo-recognition series, averaged over subjects and over trial-blocks.

against their training frequency. Each point in these curves is an average based on 120 observations ( 15 subjects, 4 trial blocks, 2 words). It is evident that there is an overall training-frequency effect, which is confirmed by a significant $F$ ratio $(F=30.71 ; d f=4,112 ; p<.001)$. The

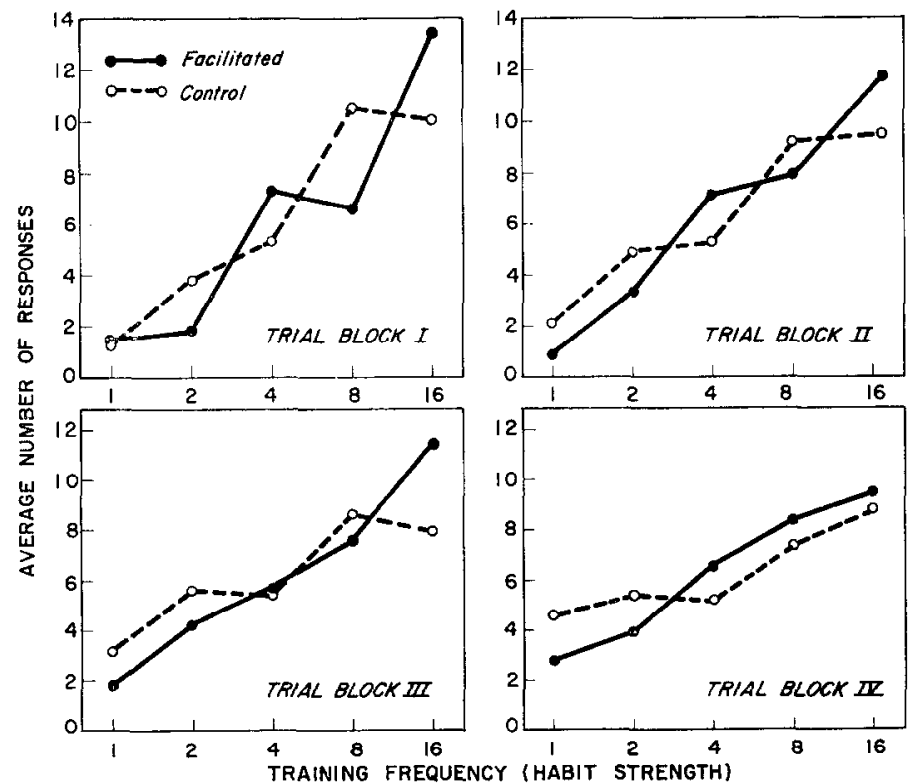

Fig. 2. Average number of responses of different frequency classes in separate trial blocks of the pseudo-recognition series. 
response emission of both groups of subjects is clearly a function of training frequency-and, apparently, a linear function of its logarithm.

Of major interest, however, is the Conditions $\times$ Frequency interaction. As predicted, responses that were highly trained were found to benefit from the presence of an audience, while responses that received minimal training were found to suffer. The appropriate interaction term in the analysis of variance was significant at the .05 level $(F=2.56$; $d f=$ 4, 112).

Figure 2 shows response emission for the four trial blocks separately. Each point in these curves is based on thirty observations (15 subjects, 2 words). We note that within each trial block weaker habits suffer and strong ones benefit from the presence of spectators. The slopes of the Facilitated group subjects are consistently stecper than those of the Control subjects. This is evident from Table 1, where the slopes of the cight curves are shown, together with the differences between them. Also seen in Table 1 is the tendency of the slopes to attenuate over the suc-

TABLE 1

Siopes of the Response-Emission Curves

\begin{tabular}{cccc}
\hline Trial block & $\mathrm{bx}$ (Control) & $\mathrm{bx}^{\prime}$ (Facilitated $)$ & $\mathrm{bx}^{\prime}-\mathrm{bx}$ \\
\hline I & .556 & .763 & .207 \\
II & .442 & .639 & .197 \\
III & .297 & .589 & .292 \\
IV & .275 & .414 & .139 \\
All trials & .308 & .669 & .361 \\
\hline
\end{tabular}

cessive trial blocks. This finding, true for both conditions, is supported by the significant interaction between Trial Blocks and Frequency $(F=$ $2.63 ; d f=12,336 ; p<.01$ ).

\section{DISCUSSION}

The predicted interaction between conditions and habit strength was obtained both in the overall results and for each trial block scparately. While the slopes of the curves of the two conditions differ in the predicted direction, there seems to be one reversal. At training frequency 8 the Control group surpasses the Facilitation group on the first three trial blocks. A systematic interaction pattern, however, is evident on the fourth trial block. The particular significance of this reversal is a matter of speculation. The habits based on 8 repetitions are apparently considerably weaker than habits acquired by 16 repetitions and are, therefore, likely to suffer from the presence of an audience. Suffice it to observe, however, 
that as predicted, the slopes of the two conditions differ for each trial block, that they intersect, and that they diverge at low- and high-training frequencies.

The overall pattern of results is quite similar to those obtained by Zajone and Nieuwenhuyse (1964). They, too, obtained a significant frequency effect, and a significant Conditions $\times$ Frequency interaction. In their experiment, it should be noted, the conditions were created by a dircet motivational manipulation.

There is some difference between the results in the two studies. Subjects in the Zajonc-Nieuwenhuyse experiment matched their pseudo-recognition responses considerably closer to the prior training frequencies than subjects in the present experiment. The slope of the curve relating overall response emission to response frequency in the Zajonc-Nieuwenhuyse experiment was 1.09 , and its origin nearly zero, indicating an almost perfect match between response and training frequencies. The slopes in the present experiment, as can be seen from Table 1, are somewhat less, and the origins of the curves higher than zero (Fig. 1). These lesser slopes mean that subjects give as guesses a greater number of infrequent and a smaller number of frequent words than they were given in training. Examination of Table 1 and Fig. 2 also shows that this tendency increases over the successive trial blocks; i.c., the slopes become less steep. The attenuation of slopes also occurs in the Zajonc-Nieuwenhuyse experiment,

This difference in results is due to the difference in the conditions of the pseudu-recognition series in the two experiments. Within each block of but this tendency is relatively weak and not significant.

41 trials there were in the present experiment ten presentations-all well-above threshold-showing once each of the ten training words. The subjects could recognize these stimuli better than $90 \%$ of the time $(92.5 \%$ in the Control and $91.3 \%$ in the Facilitated groups). Subjects were reminded, therefore, of all the words given in training and could use them later as guesses. This procedure was of particular benefit to the lowfrequency responses, since they were most readily forgotten. As a consequence of seeing these low-frequency words, the number of times they were used as guesses increased. But, since each subject had a constant number of guesses (trials), he necessarily called out a smaller number of high-frequency words. Hence, the slope of the response emission curve was reduced, and its origin elevated.

In the Zajonc-Nieuwenhuyse experiment, the real stimuli served to assess recognition threshold. Since the ascending method of limits was employcd, they were shown during the early trials under subthreshold viewing conditions. They could hardly be seen at all on the first trial block. They could not, therefore, remind the subject of the infrequent 
words which he might have forgotten. As the viewing conditions improved over the successive trial blocks, reaching and surpassing the subject's threshold, the real stimuli began having an effect on the subject's responses. And on the last trial block the slope of the response emission curve in the Zajonc-Nieuwenhuyse experiment was .583 for the combined conditions, approaching the figures for the first trial block of the present experiment."

For the purpose of verifying the hypothesis that social facilitation effeets are due to a drive-like enhancement of dominant responses, the matching of response frequencies to training frequencies is not crucial. Of major importance is the comparison of the Conditions $\times$ Frequency interactions in the two experiments, and this comparison shows a close correspondence between the two sets of results.

The question can be raised whether effects similar to those above could be obtained under the classical coaction conditions (Allport, 1924), that is, where several individuals-in the presence of one another-engage in the same task. Dashiell's (1930) results seem to show the audience effect to be more pronounced for such tasks as multiplication and chain association. Whether audience has a stronger facilitation effect than coaction in the pseudo-recognition situation is a research question which is under investigation.

\section{REFERENCES}

Allport, F. H. Social psychology. Boston: Houghton Mifflin, 1924.

Bengum, B. O., AхI LeHк, D. J. Effects of authoritarianism on vigilance performance. Journal of Applied Psychology, 1963, 47, 75-77.

Dashield, J. F. An experimental analysis of some group effects. Journal of Abnormal and Social Psychology, 1930, 25, 190-199.

Gates, M. G., AND Allee, W. C. Conditioned behavior of isolated and grouped cockroaches on a simple maze. Journal of Comparative Psychology, 1933, 13, 331-358.

HarLow, H. F. Social facilitation of feeding in the albino rat. Journal of Genetic Psychology, 1932, 41, 211-221.

Husband, R. W. Analysis of methods in human maze learning. Journal of Genetic. Psychology, 1931, 39, 258-277.

JAMES, W. T. Social facilitation of eating behavior in puppies after satiation. Joumal of Comparative and Physiological Psychology, 1953, 46, 427-428.

KLopfer, P. H. Influence of social interaction on learning rates in birds. Science, $1958,128,903$.

Mason, J. W., And Brady, J. V. The sensitivity of psycho endocrine system to social and phvsical environment. In Leiderman, P. H., and Shapiro, D. (Eds.) Psychobiological approaches to social behavior. Stanford: Stanford Univer. Press, 1964.

Pessin, J. The comparative effects of social and mechanical stimulation on memorizing. American Journal of Psychology, 1933, 45, 263-270.

"Figure 2 in the Zajonc-Nieuwenhuyse study shows response emission in percentages. For purposes of comparison with the present data, however, these slopes were computed on the absolute number of responses emitted. 
Solomon, R. L., and Postman, L. Frequency of usage as a determinant of recognition threshold for words. Journal of Experimental Psychology, 1952, 43, 195-210.

Spence, K. W. Behavior theory and conditioning. New Haven: Yale Univer. Press, 1956.

Thiesses, D. D. Population density, mouse genotype, and endocrine function in behavior. Joumal of Comparative and Physiological Psychology, 1964, 57, 412416.

Tolman, C. W., and Wilsox, G. F. Soeial feeding in domostic chieks. Animal Behavior, $1965,13,134-142$.

Travis, L. E. The effect of a small audience upon cyc-hand coordination. Journal of Abnormal and Social Psychology, 1925, 20, 142-146.

ZAJONc, R. B. Social facilitation. Science, 1965, 149, 269-274.

ZaJonc, R. B., AND NiwuwenhuYse, B. Relationship between word frequency and recognition: Perceptual process or response bias? Joumal of Experimental Psychology, 1964, 67, 276-285.

(Received September 7, 1965) 